Open Access

\title{
Adaptive mutations in sugar metabolism restore growth on glucose in a pyruvate decarboxylase negative yeast strain
}

\author{
Yiming Zhang 1,4, Guodong Liu, ${ }^{1,4}$, Martin KM Engqvist ${ }^{1,4}$, Anastasia Krivoruchko 1,4, Björn M Hallström²,
} Yun Chen ${ }^{1,4}$, Verena Siewers ${ }^{1,4}$ and Jens Nielsen ${ }^{1,3,4^{*}}$

\begin{abstract}
Background: A Saccharomyces cerevisiae strain carrying deletions in all three pyruvate decarboxylase (PDC) genes (also called Pdc negative yeast) represents a non-ethanol producing platform strain for the production of pyruvate derived biochemicals. However, it cannot grow on glucose as the sole carbon source, and requires supplementation of $\mathrm{C}_{2}$ compounds to the medium in order to meet the requirement for cytosolic acetyl-CoA for biosynthesis of fatty acids and ergosterol.

Results: In this study, a Pdc negative strain was adaptively evolved for improved growth in glucose medium via serial transfer, resulting in three independently evolved strains, which were able to grow in minimal medium containing glucose as the sole carbon source at the maximum specific rates of $0.138,0.148,0.141 \mathrm{~h}^{-1}$, respectively. Several genetic changes were identified in the evolved Pdc negative strains by genomic DNA sequencing. Among these genetic changes, 4 genes were found to carry point mutations in at least two of the evolved strains: MTH1 encoding a negative regulator of the glucose-sensing signal transduction pathway, HXT2 encoding a hexose transporter, CIT1 encoding a mitochondrial citrate synthase, and RPD3 encoding a histone deacetylase. Reverse engineering of the non-evolved Pdc negative strain through introduction of the MTH1 $1^{810}$ allele restored its growth on glucose at a maximum specific rate of $0.053 \mathrm{~h}^{-1}$ in minimal medium with $2 \%$ glucose, and the CIT1 deletion in the reverse engineered strain further increased the maximum specific growth rate to $0.069 \mathrm{~h}^{-1}$.
\end{abstract}

Conclusions: In this study, possible evolving mechanisms of Pdc negative strains on glucose were investigated by genome sequencing and reverse engineering. The non-synonymous mutations in MTH1 alleviated the glucose repression by repressing expression of several hexose transporter genes. The non-synonymous mutations in HXT2 and CIT1 may function in the presence of mutated MTH1 alleles and could be related to an altered central carbon metabolism in order to ensure production of cytosolic acetyl-CoA in the Pdc negative strain.

Keywords: Pyruvate decarboxylase, Genomic DNA sequencing, Yeast, Reverse engineering, MTH1, Hexose transporter, Citrate synthase, Histone deacetylase

\section{Background}

Saccharomyces cerevisiae is an important cell factory widely used for the production of beer, bread, wine, bioethanol, nutraceuticals, chemicals and pharmaceuticals $[1-5]$. When grown on glucose, the majority of the

\footnotetext{
*Correspondence: nielsenj@chalmers.se

${ }^{4}$ Novo Nordisk Foundation Center for Biosustainability, Chalmers

University of Technology, Kemivägen 10, 41296 Göteborg, Sweden

Full list of author information is available at the end of the article
}

glycolytic flux is directed towards ethanol due to the so-called Crabtree effect in S. cerevisiae. The only strategy to eliminate ethanol production that has worked so far is removing pyruvate decarboxylase activity so that pyruvate cannot be converted to acetaldehyde, the direct precursor of ethanol [6]. In S. cerevisiae, pyruvate decarboxylase is encoded by three structural genes, $P D C 1$, $P D C 5$ and PDC6 [7-9]. However, pdc triple deletion mutants $(p d c 1 \Delta p d c 5 \Delta p d c 6 \Delta$, also called Pdc negative 
strains) cannot grow on glucose as the sole carbon source [10]. The metabolic responses of Pdc negative strains, growth requirements, and growth recovery by threonine aldolase (encoded by GLY1) over-expression all suggested that the growth defect of Pdc negative strain on glucose was due to the lack of cytosolic acetyl-CoA for biosynthesis of cellular biomolecules, especially lipids.

Interestingly, the Pdc negative strains are still sensitive to high glucose concentrations even when supplemented with a $\mathrm{C}_{2}$ source or with GLY1 over-expression $[6,11]$. van Maris et al. performed directed evolution of a Pdc negative strain on glucose, yielding the ' $\mathrm{C}_{2}$-independent, glucose-tolerant, and pyruvate-hyperproducing' strain TAM [12]. In the TAM strain, a MTH1 allele with a 225 bp internal deletion $(M T H 1-\Delta T)$ was identified, and was attributed with restoring growth of the Pdc negative strain on glucose [13]. However, when introducing the MTH1- $\Delta T$ allele into an un-evolved Pdc negative strain, the growth rate $\left(\mu_{\max }=0.10 \mathrm{~h}^{-1}\right)$ was slower in minimal medium with $2 \%$ glucose, compared to the TAM strain $\left(\mu_{\max }=0.20 \mathrm{~h}^{-1}\right)$, indicating the possible presence of additional advantageous genetic changes in the TAM strain besides $M T H 1-\triangle T$.

Mth1 functions as a negative transcriptional regulator in the glucose signaling pathway together with other regulators, i.e. Snf3, Rgt2, Std1, Rgt1. Several other MTH1 alleles have been identified in selections of glucose or catabolite repression suppressors using glucose sensitive mutants [14-18]. The MTH1 alleles seemed to be able to resolve the sensitivity to glucose in these mutants. Previous studies have shown that these MTH1 alleles reduced glucose transport by repressing the transcription of several hexose transporter genes (HXTs) [12, 14, 16, 17], and that over-expression of MTH1 had similar effects [13]. It has been proposed that MTH1- $\Delta T$ resulted in a decreased degradation of Mth1 [13], which could be related to putative PEST sequences (usually present in proteins with short intracellular half-life) and a target site for phosphorylation by casein kinase Yck1 [19] situated inside the deleted region. Mth1 or its paralog Std1 interacts with Rgt1, which also interacts with other transcription factors and binds the promoters of hexose transporter genes [20, 21]. The decreased degradation of Mth1 could prevent the phosphorylation of Rgt1, required for its release from the promoters of several hexose transporters [21]. The decreased degradation of Mth1 resulted from the $M T H 1-\Delta T$ allele could therefore repress the transcription of hexose transporter genes even during growth on glucose.

In this study, a Pdc negative strain PDC-E1 (MATa ura3-52 his3- $\Delta 1$ pdc1 $\Delta p d c 5 \Delta p d c 6 \Delta$ ) was evolved in glucose medium via serial transfer in 3 independent culture lines. Genomic DNA sequencing of these three evolved Pdc strains identified mutations in MTH1 as well as in HXT2 [22], CIT1 [23] and RPD3 [24]. The fact that the mutated MTH1 alleles were also identified in our evolved Pdc negative strains, indicated again that MTH1 might be an important target for relieving high glucose repression. In order to understand the roles of these genetic changes in the evolved strains, the effects of the mutations in mutated genes, MTH1, HXT2, CIT1 and $R P D 3$, were investigated using homology analysis of their protein sequences and published crystal structure models of homologous proteins, and possible mechanisms were proposed and discussed. Although the speculations regarding the possible mechanisms in evolved Pdc negative strains still require further investigations, they may be useful and helpful for metabolic engineering strategies on Pdc negative strains.

\section{Results and discussion}

\section{Construction of Pdc negative strains and strain evolution}

CEN.PK 113-5D (MATa ura3-52) and CEN.PK 110-10C

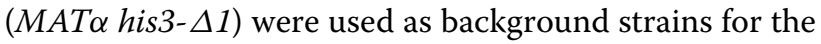
construction of Pdc negative strains (Additional file 1: Figure S1). A collection of triple deletion mutants were obtained using a bipartite deletion strategy (Additional file 1: Figure S2), strain crossings, and tetrad segregations, including PDC-E1 (MATa ura3-52 his3- $\Delta 1$ pdc1 $\Delta$ $p d c 5 \Delta p d c 6 \Delta$ ) (Table 1$)$.

PDC-E1 (MATa ura3-52 his3- 11 pdc1 $\Delta$ pdc5 $\Delta$ $p d c 6 \Delta$ ), E1 for short, was evolved in 3 independent culture lines in YPD medium with gradually reduced

\section{Table 1 Strains used in this study}

\begin{tabular}{|c|c|}
\hline Strain name & Genotype or description \\
\hline CEN.PK 113-5D & MATa ura3-52 \\
\hline CEN.PK 110-10C & MAT $\alpha$ his3- $\Delta 1$ \\
\hline PDC-A1 & MATa ura3-52 pdc5 $\Delta$ \\
\hline PDC-A2 & MATa ura3-52 pdc1 $\Delta$ \\
\hline PDC-A3 & MATa ura3-52 pdc6 $\Delta$ \\
\hline PDC-B1 & MATa ura3-52 pdc1 $\Delta$ pdc5 $\Delta$ \\
\hline PDC-B2 & MAT $\alpha$ ura3-52 pdc1 $\Delta$ pdc6 $\Delta$ \\
\hline PDC-C1 & MATa ura3-52 pdc1 $\Delta p d c 5 \Delta p d c 6 \Delta$ \\
\hline PDC-C2 & MAT $\alpha$ ura3-52pdc1 $\Delta p d c 5 \Delta p d c 6 \Delta$ \\
\hline PDC-D1 & MATa his3- $\Delta 1 p d c 1 \Delta p d c 5 \Delta p d c 6 \Delta$ \\
\hline PDC-D2 & MAT $\alpha$ his3- $\Delta 1 p d c 1 \Delta p d c 5 \Delta p d c 6 \Delta$ \\
\hline PDC-E1 & MATa ura3-52 his3- $\Delta 1 p d c 1 \Delta p d c 5 \Delta p d c 6 \Delta$ \\
\hline PDC-E2 & MATa ura3-52 his3- $\Delta 1$ pdc1 $\Delta$ pdc5 $\Delta$ pdc6 $\Delta$ \\
\hline PDC-E1A & PDC-E1 adaptively evolved in glucose \\
\hline PDC-E1B & PDC-E1 adaptively evolved in glucose \\
\hline PDC-E1C & PDC-E1 adaptively evolved in glucose \\
\hline M81-11 & PDC-E1, mth1::MTH1 ${ }^{81 D}$ \\
\hline M81C & M81-11, cit1::amdSYM \\
\hline
\end{tabular}


ethanol concentration. Once fast-growing, glucose tolerant strains were obtained in YPD media, they were further evolved for $\mathrm{C}_{2}$ carbon source-independent and faster growth in minimal medium with $2 \%$ glucose.

At the end of the adaptive evolution, three independently evolved E1 strains were obtained. We refer to these as PDC-E1A, PDC-E1B, and PDC-E1C (E1A, E1B, $\mathrm{E} 1 \mathrm{C}$ for short, respectively). E1A, E1B, E1C could grow in minimal medium with glucose as the sole carbon source, with maximum specific growth rates of $0.138,0.148$, $0.141 \mathrm{~h}^{-1}$, respectively (Fig. 1).

\section{Genomic sequencing results of the unevolved and evolved Pdc negative strains}

Genome sequencing of the parental strain E1 and its evolved strains (E1A, E1B, E1C) was performed to study the genetic changes that occurred during the adaptive evolution. The raw sequencing data were filtered and trimmed to remove adaptor sequences and sequence ends with a quality score below 20 . The filtered reads

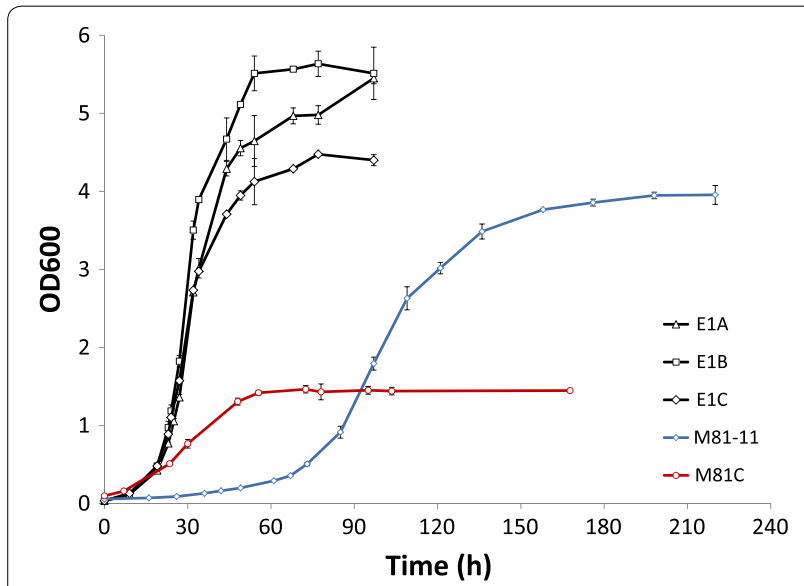

Fig. 1 Growth profiles of the evolved Pdc negative strains and reverse engineered strains M81-11 and M81C. E1A, E1B and E1C are three evolved Pdc negative strains. M81-11 is a Pdc negative strain with a point mutation in MTH1 (ura3-52 his3- $\Delta 1$ pdc1 $\Delta$ pdc5 $\Delta$ pdc6 $\Delta$ mth $1:: \mathrm{MTH}^{81 D}$ ). M81C is a M81-11 mutant with the CIT1 deletion. The cultivations were performed in minimal medium with $2 \%$ glucose in replicate. Error bars represent \pm standard errors. were mapped to the CEN.PK 113-7D reference genome. In the E1A strain, three single nucleotide variants (SNVs) in coding regions representing non-synonymous mutations were identified, as listed in Additional file 2: Table $\mathrm{S} 2$. In the E1B strain, 11 SNVs in coding regions representing non-synonymous mutations were identified. In the E1C strain, six SNVs in coding regions representing non-synonymous mutations, one chromosomal regional deletion, one mitochondrial regional deletion and one single nucleotide insertion were identified.

Among all genes with SNVs, three genes, MTH1, CIT1, $H X T 2$, were found to carry point mutations in all three evolved strains. And one gene, RPD3, was found to carry point mutations in two of the evolved strains. The mutations of the proteins encoded by the four genes are listed in Table 2. It is interesting to note that the same mutations occurred in more than one strain for two loci, A81D in Mth1 and W466* in Hxt2, and that two mutations affected adjacent amino acids, P176Q and H175R in Cit1.

\section{Integration of $M T H 1^{81 D}$ in non-evolved Pdc negative strain E1}

As previously reported by Oud et al. [13], a 225 bp inframe internal deletion (corresponding to amino acids 57-131) in MTH1 was identified in their evolved Pdc negative strain TAM, which was demonstrated to be responsible for relieving glucose sensitivity in the Pdc negative mutant. Among the previously identified $M T H 1$ alleles affecting glucose sensing, e.g. BPC1-1, DGT11, HTR1-5, HTR1-19 and HTR1-23, two mutations in codon 85 (I85N, I85S) and one mutation in codon 102 (S102G) were found, and it was also found that the S102G mutation could reinforce the mutations in codon 85 although having no effects by itself $[16,17]$. Since the $M T H 1^{85 S}$ allele has already been confirmed to suppress glucose repression, we chose to validate the $M T H 1^{81 D}$ allele through reverse engineering in this study, to investigate if it has similar effects of suppressing glucose repression like other alleles.

The $M T H 1^{81 D}$ allele was integrated into the MTH1 locus of the non-evolved E1 strain, resulting in strain M81-11. The growth profile in minimal medium with

Table 2 Point mutations in evolved E1 strains

\begin{tabular}{|c|c|c|c|c|}
\hline \multirow[t]{2}{*}{ Name } & \multirow[t]{2}{*}{ Description } & \multicolumn{3}{|c|}{ Evolved strains } \\
\hline & & E1A & E1B & E1C \\
\hline Mth1 & Negative regulator of the glucose-sensing signal transduction pathway & A81/D & $185 / S$ & A81/D \\
\hline Cit1 & Mitochondrial citrate synthase & $\mathrm{P} 176 / \mathrm{Q}$ & M84/N & $\mathrm{H} 175 / \mathrm{R}$ \\
\hline $\mathrm{Hxt} 2$ & High-affinity glucose transporter of the major facilitator superfamily & W466/* & G75/R & W466/* \\
\hline Rpd3 & Histone deacetylase, component of both the Rpd3S and Rpd3L complexes & & $85 F / I$ & 196AN \\
\hline
\end{tabular}


$2 \%$ glucose is shown in Fig. 1 with the maximum specific growth rate of $0.053 \mathrm{~h}^{-1}$. The fact that $M T H 1^{81 D}$ by itself could restore the growth of the Pdc negative strain on glucose suggested it had the similar effects on glucose repression alleviation. However, the maximum specific growth rate was not as high as those of the evolved strains E1A, E1B, E1C. The $M T H 1^{81 D}$ mutation contributes around $35 \%$ of the maximum specific growth rate in the evolved E1 strains, indicating there are likely other genetic changes that contribute to their growth recovery.

Analysis of the mutations in Mth 1 and their possible effects Even though the N-terminal part of Mth1 appears to be important in glucose repression it is not yet clear why. To gain sequence-function insight into Mth1 protein, a bioinformatics approach was undertaken.

Alignment results of Mth1 from S. cerevisiae and 22 other homologous sequences of Mth1 and Std1 from unicellular fungi revealed that A81 and I85 are positioned on an 'island' composed of 22 highly conserved amino acids from codon 71-91 (Fig. 2a). Since no protein crystal structures of Mth1 or homologs thereof are available, a secondary structure prediction was performed for the conserved 'island' using four prediction tools [25] (Fig. 2b), which indicated an alpha amphipathic helix in this region with the hydrophilic side facing solvent and the hydrophobic side facing the core of the protein (Fig. 2c). The putative helix is likely initiated by the structurally rigid prolines at codon 74 and 75, and Y77, A81, I85 and L89 are buried away from the solvent based on two prediction tools [26] (Fig. 2b). Therefore, it is possible that A81 and I85, together with Y77 and L89, play a structural role in anchoring a highly conserved alpha helix to the Mth1 surface through hydrophobic interactions. The A81D, I85S or I85N mutations, representing non-polar to polar amino acid changes, would disrupt these interactions and may therefore cause structural changes in the protein.

It is interesting to note that another conserved 'island' from codon 118-137 is the identified target region for phosphorylation by Yck1 [19]. Therefore, it is reasonable to speculate that the predicted helix structure within the conserved island from codon 71-91 might play an important part in the function of Mth1.

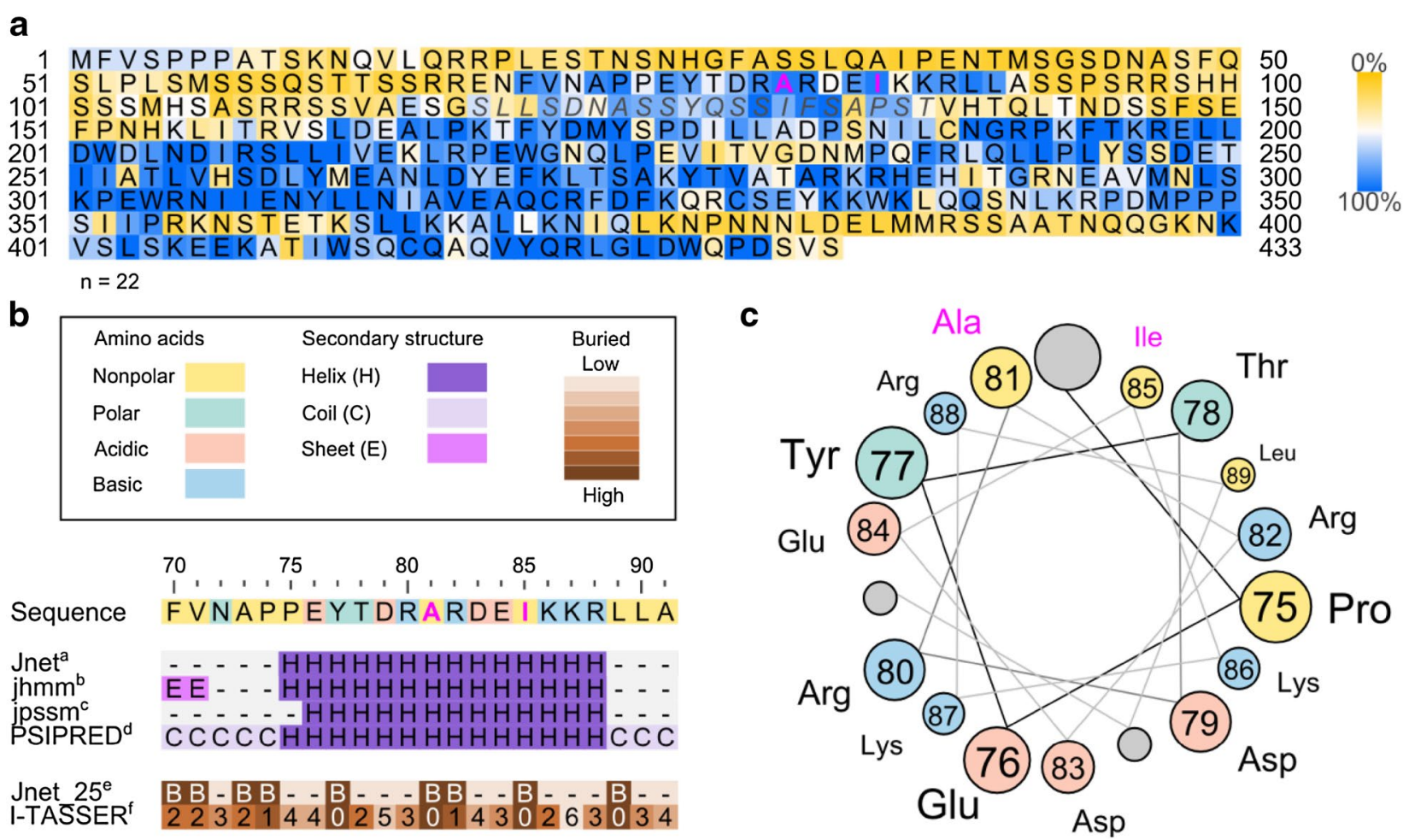

Fig. 2 Mapping and analysis of Mth1 mutations. Magenta text indicates positions for non-synonymous mutations identified in this study. a Result of a multiple alignment using homologous sequences $(n=22)$. The Mth1 sequence of S. cerevisiae is shown with colored conservation levels. Yellow indicates low conservation, white intermediate, and blue high. Gray and italic text indicates the phosphorylation region of casein kinase Yck1. b Analysis of the 22 amino acids from the conserved region at position 70-91 and secondary structure predictions using six different prediction programs $[25,26]$. c A helix wheel representation of amino acids predicted to form an alpha-helix (codon 75-89). The amino acid types are colored as in $\mathbf{b}$. 
Transcriptional analysis of hexose transporter genes

In order to understand the effects of the $M T H 1^{81 D}$ allele on transcription of genes encoding hexose transporters, transcription analysis were performed on HXT1-7 using qPCR in reverse engineered strain M81-11 and wild type strain CEN.PK 113-11C [27].

Compared with wild type strain, the expression levels of HXT1, HXT3, HXT4 and HXT6E7 were much lower in M81-11, i.e. around 9 fold, 25 fold, 15 fold, and 40 fold lower, respectively (Fig. 3). The expression level of HXT5 did not differ much between the wild type strain and the M81-11. However, the expression level of $H X T 2$ was around 3 fold higher in the M81-11 strain, which was quite a different pattern from the other $H X T \mathrm{~s}$. The different expression patterns of $H X T \mathrm{~s}$ in M81-11 is consistent with those in the TAM strain [12], suggesting that they are regulated by Mth1 differently, which is consistent with earlier findings [28].

According to the bioinformatics analysis and its homolog protein structure, the mutations identified in $\mathrm{Hxt} 2$ in evolved Pdc strains were predicted to impair or completely abolish its activity to transport glucose due to the structural changes around the transport channel (Additional file 3: Figure S5). Therefore, it is possible that the glucose transport by Hxt2 was decreased due to the identified mutations, even with an increase in its transcription level, which resulted from the mutations in Mth1.

\section{Deletion of CIT1 in the M81-11 strain}

A recent study revealed that cytosolic $\mathrm{C}_{2}$ can be derived from mitochondrial $\mathrm{C}_{2}$ in the form of acetate involving mitochondrial CoA transferase Ach1 in Pdc negative strains [29]. The Cit1 mutations might be connected with cytosolic $\mathrm{C}_{2}$ supply, since Cit1 competes with Ach1 for mitochondrial acetyl-CoA (Fig. 4). The mutated Cit1 has

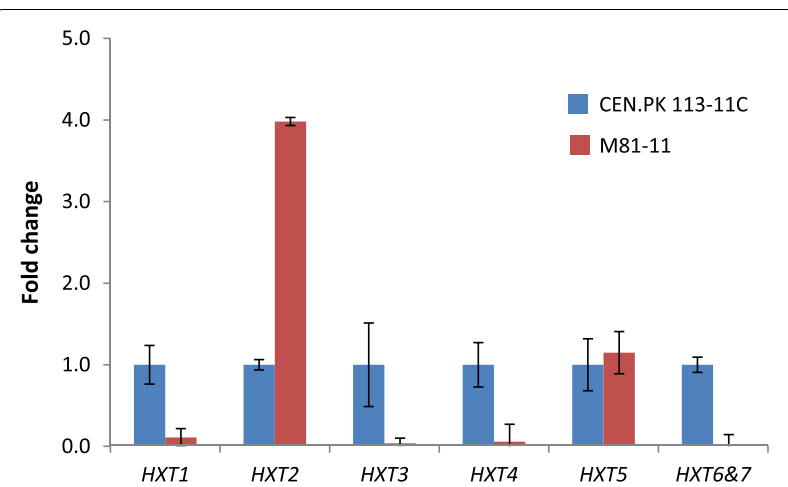

Fig. 3 Transcription analysis of HXTS (HXT1-7) in the M81-11 and wild type strain CEN.PK 113-11C. Cells for transcription analysis were harvested at exponential phase $\left(\mathrm{OD}_{600} \sim 1\right)$. The expression levels of HXTs in wild type strain were set as 1. potentially decreased activity, predicted by the analysis concerning possible structural disruptions of the identified mutations (Additional file 3: Figure S6), and this might allow more acetyl-CoA being converted to acetate by Ach1, thus providing more acetate for acetyl-CoA biosynthesis in the cytosol.

To test the hypothesis, CIT1 was deleted in the M81-11 strain, resulting in the strain M81C. Compared to M8111 , the strain M81C showed a higher maximum specific growth rate of $0.069 \mathrm{~h}^{-1}$ when cultured in minimal glucose medium (Fig. 1), indicating the CIT1 deletion did have a positive effect on cell growth. When CIT1 was deleted, the incomplete TCA cycle resulted in inefficient pyruvate dissimilation, and the accumulated pyruvate (data not shown) caused the low $\mathrm{pH}(<3)$ in the cell culture. Therefore, a lower final $\mathrm{OD}_{600}$ was observed in M81C compared to M81 (Fig. 1).

\section{Possible evolving mechanisms in Pdc negative strains}

According to the bioinformatics analysis of the point mutations on their corresponding proteins (Additional file 3), all the point mutations occurred at the highly conserved residues and might cause structural disruptions and result in enzyme activity decreases or complete activity abolishment. Therefore, possible mechanisms were proposed for how the evolved Pdc negative strains have acquired a faster growth phenotype.

The growth defect of a Pdc negative strain on glucose [10] was previously attributed to the lack of cytosolic acetyl units, since acetyl-CoA cannot be transported between different subcellular compartments freely and ethanol or acetate supplementation or GLY1 overexpression could restore its growth on glucose $[6,11$, 30]. However, the growth recovery of the Pdc negative strain with $M T H 1-\Delta T$, which does not in itself lead to cytosolic acetyl-CoA provision, indicated the presence of native pathways to cytosolic $\mathrm{C}_{2}$ compounds. This was recently identified in the form of acetate, converted from mitochondrial acetyl-CoA by CoA transferase (or acetyl-CoA hydrolase, encoded by $A C H 1$ ) [29]. Although Ach1 is involved in channeling acetyl-CoA from the mitochondria to the cytosol, it is only functional under glucose derepressed conditions and this route can also be blocked by limited acetyl-CoA availability due to the stringent regulation of the PDH complex in S. cerevisiae (Fig. 4a), e.g. via post-transcriptional phosphorylation of the Pda1 subunits [31] or transcriptional repression of the Lpd1 subunits [32]. In the evolved Pdc negative strains, the mutations in Mth1 seemed to play the most critical role, since the single mutation $M T H 1^{81 D}$ alone was here shown to improve the growth of a Pdc negative strain on glucose as well as the earlier reported truncation MTH1- $\triangle T$. According to homology analysis of Mth1 

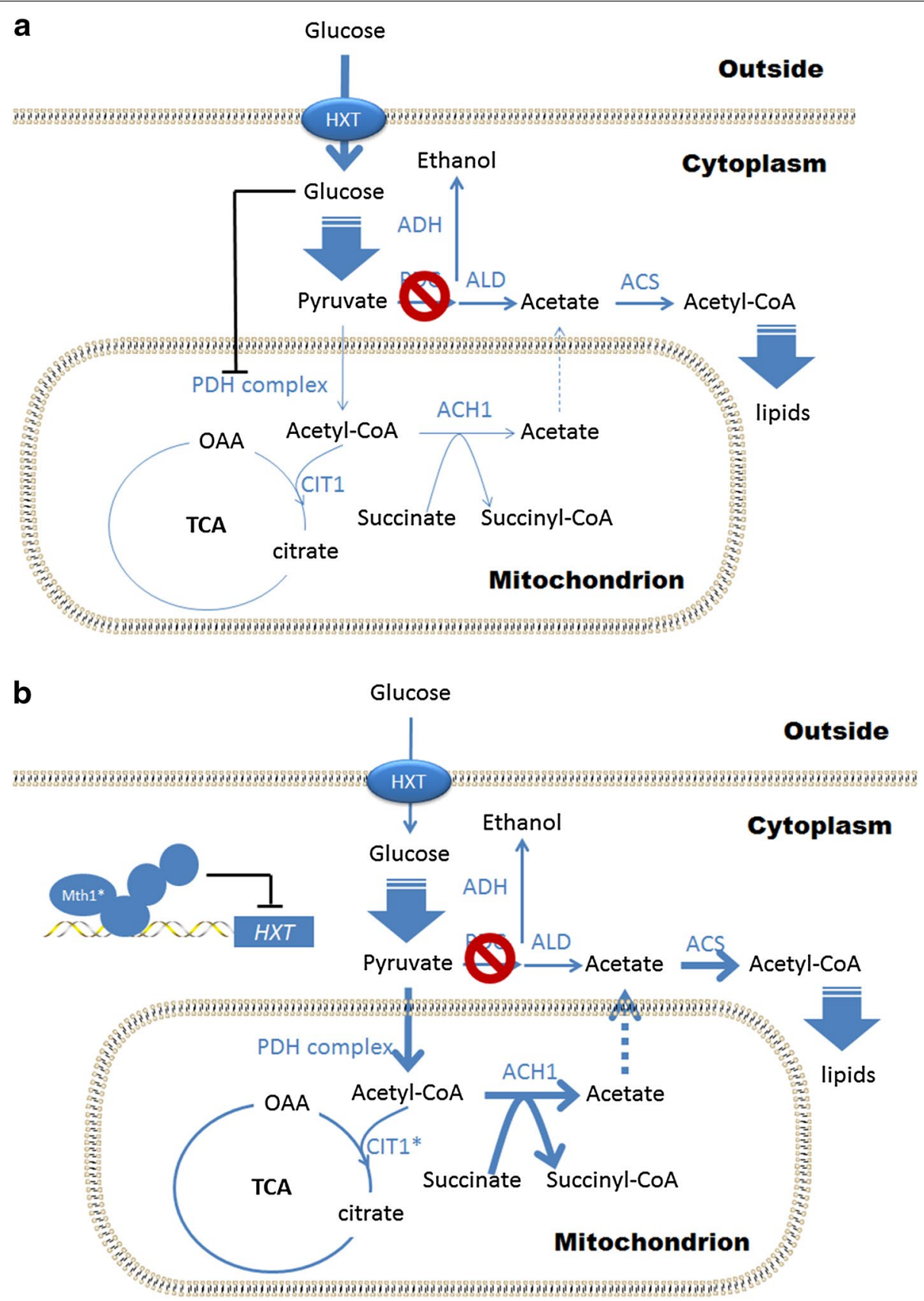

Fig. 4 A simple illustration for possible roles of mutated proteins in the evolved Pdc negative strains. The blue solid arrows represent the reactions catalyzed by the enzymes, which are indicted in blue text. The blue dash line represents the transportation between different subcellular organelles. The black lines with a bar at one end represent the repression or inhibition. The red circles represent the block due to Pdc deletions. a Simplified acetyl-CoA metabolism in the parental Pdc negative strain. The PDH complex and TCA cycle enzymes is repressed by high glucose uptake via hexose transporters (HXT). b Simplified acetyl-CoA metabolism in evolved Pdc negative strain with point mutated Mth1 (Mth $\left.1^{*}\right)$ and Cit1 (Cit $\left.1^{*}\right)$. Glucose uptake via HXT decreases in the presence of Mth $1^{*}$, resulting in derepression of the PDH complex and TCA cycle enzymes. Cit $1{ }^{*}$ with predicted decreased activity allows more mitochondrial acetyl-CoA convert to acetate by Ach1, which can be transported to the cytosol and converted to acetyl-CoA there.

and predictions of the conserved 'island' (codon 71-91), where the mutations are located, A81 and I85 may play a critical role in maintaining the alpha helix structure formed within it, and thus probably affect the function of Mth1. However, a crystal structure of Mth1 would be needed to validate these predictions. 
The MTH1 alleles seem to decrease glucose uptake transport by alleviated repression of transcribing several HXTs, especially $H X T 1$ and $H X T 3$ [13-18], which was also found in the TAM strain (evolved Pdc negative strain, with $M T H 1-\Delta T$ allele) by van Maris et al. [12]. Moreover, a deletion within HXT3 ( 1,000 bp) was also found in the evolved strain E1C, which would undoubtedly destroy its activity to transport glucose. A previous study suggested that the rate of glucose transport determines the strength of glucose repression [33], and the attenuated glucose uptake therefore likely resulted in a generally reduced glucose repression in the evolved strains. Thus, the transcription of many genes, which are normally repressed by glucose, could probably be partially de-repressed despite the high extracellular glucose concentration, e.g. genes encoding mitochondrial enzymes. Therefore, in the evolved strains with mutated $M T H 1$, it is possible that the $\mathrm{C}_{2}$ supply from the mitochondria to the cytosol via Ach1 route was no longer blocked (Fig. 4b).

Hxt2, a high-affinity glucose transporter, is usually found to function under low glucose concentrations and its transcription is repressed by high glucose and induced by low glucose $[34,35]$. The mutations in Hxt2 seemed to make no sense in high glucose medium ( $2 \%$ glucose) used in this study. One possible hypothesis would be that the effects of the mutated Mth1 might be quite complicated. Transcriptional analysis of the TAM strain showed decreased transcription of HXT1, HXT3, HXT4, HXT6 and $H X T 7$, and increased transcription of $H X T 2$ and $H X T 5$, although this might resulted from other genetic changes besides $M T H 1-\Delta T$ [12]. Previous studies with other $M T H 1$ alleles revealed significant decreased transcription of $H X T 1$ and $H X T 3$, a large increase in $H X T 7$ and nearly no change in $H X T 2$ [16, 17]. All these results suggested that MTH1 alleles might have different effects in regulating expression of hexose transporters with low affinity compared to those with high affinity. According to previous results, the mutated $M T H 1$ might result in an unchanged or increased transcription level of $H X T 2$, which has been confirmed by our qPCR results (Fig. 3). The mutations in Hxt2 might cause structural disruptions based on our predictions (Additional file 3: Figure S5), and may therefore further reduce the glucose transport.

As mentioned above, the role of the mutated Cit1 might also be connected with cytosolic $\mathrm{C}_{2}$ supply via the mitochondria. Since $C_{2}$ carbon supply in the cytosol seemed to be a limiting step for the growth of Pdc negative strains [29]. The mutated Cit1 with decreased activity might further improve the strain growth on glucose in the presence of the mutated Mth1, since Cit1 competes with Ach1 for mitochondrial acetyl-CoA. The complete disruption of CIT1 in the M81-11 strain increased the maximum specific growth rate, which supports this speculation.

Rpd3 usually functions in the form of a complex together with other proteins to regulate gene transcription, silencing and many other processes by histone deacetylation [36-38]. More and more studies suggest that histone acetylation and deacetylation regulate gene transcription in complex and comprehensive ways [39]. Although the mutations in Rpd3 might result in its decreased activity due to the possible structural disruptions (Additional file 3: Figure S7), it is still difficult to speculate about their role in the evolved strains. A previous study found that histone acetylation and deacetylation was directly regulated by nucleocytosolic acetyl-CoA abundance [40]. One possible speculation would be that the Rpd3 mutations might be related to cytosolic acetyl-CoA abundance, but this will require further investigation.

\section{Conclusions}

In this study, a Pdc negative strain was adaptively evolved in glucose media via serial transfer, and evolved Pdc negative strains were shown to grow on glucose as the sole carbon source. Genomic DNA sequencing results of the parental Pdc negative strain and its corresponding evolved strains revealed four genes which carried point mutations in at least two of the evolved strains. The mutations in these four genes seemed to be related to the cytosolic acetyl-CoA supply. These findings will be useful for the fundamental understanding of acetyl-CoA metabolism in S. cerevisiae, as well as strain development for biochemical production as cell factories.

\section{Methods}

\section{Strain construction}

PDC1, PDC5 and PDC6 were deleted using a bipartite strategy [41] (Additional file 1: Figure S1). Sequences upstream and downstream of the individual genes were amplified using primers 1-12 listed in Additional file 1: Table S1. Two overlapping fragments of the $\operatorname{kanMX}$ resistance marker cassette flanked by loxP sites were PCR amplified from plasmid pUG6 [42] using primers 13-16 listed in Additional file 1: Table S1. The two fused PCR fragments for each gene deletion were transformed into yeast using the lithium acetate method [43]. After each gene deletion, the kanMX marker cassette was looped out via Cre recombinase mediated recombination between the two flanking loxP sites using plasmid pUC47 or pUG62 as described previously [42]. Each gene deletion was confirmed using primers 17-22 listed in Additional file 1: Table $\mathrm{S} 1$.

PDC1, PDC5, and PDC6 were consecutively deleted in two different background strains, CEN.PK 113-5D 
(MATa ura3-52) and CEN.PK 110-10C (MATa his3- 41$)$ [27]. Together with strain crossing and tetrad segregation, a collection of triple deletion mutants was constructed carrying different auxotrophic markers: ura3-52, his3- $\Delta 1$ or ura3-52 his3- $\Delta 1$. The mating type of these pdc negative mutants was determined using primers $35-37$ listed in Additional file 1: Table S1.

To create a Pdc-negative strain with a point mutation in $M T H 1$, an $M T H 1^{81 D}$ construct was created and used to replace the normal $M T H 1$ gene in strain PDC-E1 (MATa ura3-52 his3- $\Delta 1 p d c 1 \Delta p d c 5 \Delta p d c 6 \Delta$ ), as shown in Additional file 1: Figure S3, resulting in the M81-11. $M T H 1^{81 D}$ with upstream and downstream sequences was amplified using primers $23-26$, and the sequence downstream of $M T H 1^{81 D}$ was amplified using primers 27-28. Two overlapping fragments of the amdSYM marker cassette were PCR amplified from plasmid pUG-amdSYM (obtained from Euroscarf, Accession Number: P30669) using primers 29-32. The two fused PCR fragments for integration were transformed into PDC-E1 using the electroporation method as described in [44]. The integration of $M T H 1^{81 D}$ and amdSYM was confirmed using primers 33-34. The amdSYM marker was looped out via selection on counter-selective plates. The replacement of $M T H 1^{81 D}$ was confirmed by sequencing PCR product amplified by primers $33-34$.

CIT1 was deleted in strain M81-11 using the same strategy as described in $P D C 1$ deletion (Additional file 1: Figure S2), in which the amdSYM marker was used instead of the $k a n M X$ marker, resulting the strain M81C. Sequences upstream and downstream of the individual genes were amplified using primers 38-41 listed in Additional file 1: Table S1. Two overlapping fragments of the amdSYM marker cassette were PCR amplified from plasmid pUG-amdSYM using primers 29-32 listed in Additional file 1: Table S1. The two fused PCR fragments for integration were transformed into M81-11 using the electroporation method as described in [44]. The gene deletion was confirmed using primers $42-43$ listed in Additional file 1: Table S1.

\section{Medium and culture conditions}

Cultivations were performed at $30^{\circ} \mathrm{C}$ in YP medium with $2 \%$ glucose (YPD) or $2 \%$ ethanol (YPE). Selections for transformants containing the $\operatorname{kan} M X$ marker were performed on YPD or YPE plates supplemented with $200 \mathrm{mg} / \mathrm{L}$ G418 sulfate (Formedium Ltd., Hunstanton, UK). Selections for transformants carrying the amdSYM marker were performed on SM-Ac plates with $2 \%$ ethanol instead of glucose, and the counter selections were performed on SM-Fac plates with ethanol as carbon source, as described in [45]. A diploid resulting from two haploids of different mating type was obtained on agar plates containing a synthetic medium consisting of yeast nitrogen base (Formedium Ltd.), complete supplement mixture w/o uracil or histidine (Formedium Ltd.), and 2\% $(\mathrm{v} / \mathrm{v})$ ethanol (SE-ura-his). Tetrad dissections were performed on YPE agar plates.

Cultivations for strain characterization were performed at $30^{\circ} \mathrm{C}$ in triplicate in $100 \mathrm{~mL}$ shake flasks with $40 \mathrm{~mL}$ defined minimal medium. The defined minimal medium for cultivation was composed of $20 \mathrm{~g} / \mathrm{L}$ glucose, $7.5 \mathrm{~g} / \mathrm{L}$ $\left(\mathrm{NH}_{4}\right)_{2} \mathrm{SO}_{4}, 14.4 \mathrm{~g} / \mathrm{L} \quad \mathrm{KH}_{2} \mathrm{PO}_{4}, 0.5 \mathrm{~g} / \mathrm{L} \mathrm{MgSO}_{4} \cdot 7 \mathrm{H}_{2} \mathrm{O}$, $2 \mathrm{~mL} / \mathrm{L}$ trace metal solution, $1 \mathrm{~mL} / \mathrm{L}$ vitamin solution, with the $\mathrm{pH}$ adjusted to 6.5 by adding $2 \mathrm{M} \mathrm{NaOH}$. The final concentrations of trace metal elements and vitamins were previously described in [46]. The minimal medium was supplemented with $40 \mathrm{mg} / \mathrm{L}$ uracil or $40 \mathrm{mg} / \mathrm{L}$ histidine when required.

\section{Determination of biomass and extracellular metabolites}

Biomass was determined by optical density $\left(\mathrm{OD}_{600}\right)$ measurement at a wavelength of $600 \mathrm{~nm}$ with a GENESYS ${ }^{\mathrm{TM}}$ 20 Visible spectrophotometer (Thermo Electron Scientific, Madison, USA). Glucose, ethanol, glycerol, pyruvate and formate concentrations were determined in culture supernatants by high-performance liquid chromatography (Dionex-HPLC, Sunnyvale, CA, USA) equipped with UV detector and RI detector using a Bio-Rad HPX 87H column (Bio-Rad, Hercules, CA, USA). The HPLC was operated at $45^{\circ} \mathrm{C}$ with $5 \mathrm{mM} \mathrm{H}_{2} \mathrm{SO}_{4}$ as mobile phase at a flow rate of $0.6 \mathrm{~mL} \mathrm{~min}^{-1}$.

\section{Adaptive evolution of Pdc negative strain in glucose medium via serial transfer}

The adaptive evolution of PDC-E1 (MATa ura3-52 his3- $\Delta 1 p d c 1 \Delta p d c 5 \Delta p d c 6 \Delta$ ) towards growth on glucose as the sole carbon source were performed in three independent culture lines in $100 \mathrm{~mL}$ shake flasks with $20 \mathrm{~mL}$ medium at $30^{\circ} \mathrm{C}$, which involved three phases (Additional file 1: Figure S4). In the first phase, strains were cultivated in YP medium containing $1.4 \%$ glucose and $0.6 \%$ ethanol and then serially transferred every 48 or $24 \mathrm{~h}$ using YP medium with gradually decreased ethanol concentration for 8 days. Subsequently, the strains were evolved in YPD medium for 15 days and transferred with passage every 48 or $24 \mathrm{~h}$. Finally the strains were transferred into minimal medium containing $2 \%$ glucose as the sole carbon source and evolved for increased growth by serial transfer every 48 or $24 \mathrm{~h}$ for 39 days. And single clone isolates were obtained from the last shake flasks, and designated as PDC-E1A, PDC-E1B, and PDC-E1C.

\section{Genomic DNA extraction and sequencing procedures}

PDC-E1 and their evolved strains were cultured in $10 \mathrm{~mL}$ YPE medium at $30^{\circ} \mathrm{C}$, and cells were harvested 
during exponential phase for genomic DNA extraction. Genomic DNA was extracted using the Genomic DNA buffer set (QIAGEN, Hilden, Germany) and QIAGEN Genomic-tip 500/G. The DNA samples were prepared for sequencing using the Illumina DNA TruSeq protocol, with an insert size of $650 \mathrm{bp}$. The sequencing was performed multiplexed on an Illumina MiSeq using the version 2 chemistry $(2 \times 250 \mathrm{bp}$, paired reads).

\section{Analysis of genome sequencing results}

The raw sequencing data was filtered and trimmed to remove adaptor sequences and sequence ends with a quality score below 20 . The filtered reads were mapped to the CEN.PK 113-7D reference genome (http://cenpk. tudelft.nl) using the mapper MosiakAligner version 2.1.32 (http://code.google.com/p/mosaik-aligner/) with a hash size of 15 . Sites with potential indels were detected and realigned using the tools from the Genome Analysis Toolkit (GATK, version 2.3.9) [47], RealignerTargetCreator and IndelRealigner and potential PCR duplicates were eliminated using the MarkDuplicates tool from Picard version 1.100. (http://picard.sourceforge.net). The number of mappable reads after post-processing ranged from 2.9 to 5.2 million resulting in a mapped coverage spanning from approximately $50 \times$ to over $100 \times$ in the different samples. Variant calling (single nucleotide variants and small indels) was performed using GATK UnifiedGenotyper, and annotation of the detected variants was performed using SnpEff version 3.4 (http://snpeff. sourceforge.net). Finally, the alignments were visually inspected for all detected variants, in order to eliminate obvious false positives caused by incorrect alignments.

\section{qRT-PCR procedures and gene expression analysis}

Cells were cultured in shake flasks using minimal medium with $2 \%$ glucose in triplicate, and harvested for gene expression analysis at exponential growth phase $\left(\mathrm{OD}_{600} \sim 1.0\right)$ by centrifugation at $-20^{\circ} \mathrm{C}$, quenched by liquid nitrogen, and stored at $-80^{\circ} \mathrm{C}$ for use. Total RNA was isolated using RNeasy Mini Kit (QIAGEN), which was then processed to obtain fragmented cDNA using a QuantiTect Reverse Transcription Kit (QIAGEN). $2 \mu \mathrm{L}$ of the synthesized cDNA (corresponding to 100 ng RNA) was used as the template for the qPCR reaction to a final reaction volume of $20 \mu \mathrm{L}$, using a DyNAmo Flash SYBR Green qPCR Kit (Thermo Scientific, USA). Quantitative RT-PCR was performed on Stratagene Mx3005P (Agilent Technologies, USA). The thermocycling program consisted of one hold at $95^{\circ} \mathrm{C}$ for $15 \mathrm{~min}$, followed by 40 cycles of $10 \mathrm{~s}$ at $95^{\circ} \mathrm{C}$ and $20 \mathrm{~s}$ at $60^{\circ} \mathrm{C}$, and a final cycle of $1 \mathrm{~min}$ at $95^{\circ} \mathrm{C}, 30 \mathrm{~s}$ at $55^{\circ} \mathrm{C}$ and $30 \mathrm{~s}$ at $95^{\circ} \mathrm{C}$.

Primers for real-time PCR (Additional file 1: Table S1) were designed using Primer3 (http://bioinfo.ut.ee/ primer3-0.4.0/) with melting temperature $\left(\mathrm{T}_{\mathrm{m}}\right)$ around $60^{\circ} \mathrm{C}$. ACT1, a housekeeping gene, was selected as the reference gene [48]. Final concentration of primers used was $0.5 \mathrm{mM}$ in $20 \mu \mathrm{L}$ qPCR reactions. Due to nearly identical sequences of HXT6 and HXT7, they used the same pair of primers for real-time PCR. Therefore, the transcription analysis result for HXT6 and HXT7 was their sum-up.

\section{Bioinformatics analysis}

The protein sequence of each of the four S. cereviciae proteins Cit1 (UniProt\# P00890), Hxt2 (UniProt\# P23585), Rpd3 (UniProt\# P32561) and Mth1 (UniProt\# P35198) was separately used to query the NCBI database with the basic local alignment search tool (BLAST). To eliminate sequences with low identity to the $S$. cerevisiae sequences, the BLAST results were filtered to retain only those with more than $40 \%$ identity to the S. cerevisiae enzymes. The $40 \%$ cutoff was arbitrary set and is intended to limit the analysis to sequences which can reasonably be said to have the same function as the S. cerevisiae enzyme. Sequences were also filtered such that anything more than $95 \%$ identical to anything else in the dataset was removed. This was done to reduce bias, should there be a large number of deposited sequences of the same gene from the same organism. The remaining sequences were aligned using MUSCLE [49]. The resulting alignment was used to compute the conservation of each amino acid in the $S$. cerevisiae proteins compared to the filtered BLAST results. The computed conservation values were used to color-code sequence representations of the S. cerevisiae proteins. Homology models were generated for three of the four proteins using the Swiss-Model repository [50]. For Mth1 no homology model could be made as there is no crystal structure of a homologous protein. An alternative approach was therefore used where the secondary structure of the mutated region was predicted. This prediction was used in the data analysis.

\section{Additional files}

Additional file 1. Primers and strategies for Pdc negative strain constructions and strain evolution.

Additional file 2. Genome sequencing results of $\mathrm{Pdc}$ negative strain $\mathrm{E} 1$ and its evolved strains E1A, E1B, E1C.

Additional file 3. Bioinformatics analysis of the mutations in $\mathrm{Hxt2}$, Cit1 and Rpd3.

\section{Authors' contributions}

$Y Z$ designed and carried out the strain constructions, strain evolution, the cultivation experiments, analyzed the results and drafted the manuscript. GL performed the cultivation experiments, analyzed the results and revised the manuscript. ME performed the bioinformatics analysis on the mutations and 
revised the manuscript. $\mathrm{BMH}$ performed the genome sequencing analysis, and revised the manuscript. AK, YC, VS and JN supervised the design, revised the manuscript and coordinated the study. All authors read and approved the final manuscript.

\section{Author details}

${ }^{1}$ Department of Biology and Biological Engineering, Chalmers University of Technology, Kemivägen 10, 41296 Göteborg, Sweden. ${ }^{2}$ Science for Life Laboratory, KTH-Royal Institute of Technology, 17121 Stockholm, Sweden. ${ }^{3}$ Novo Nordisk Foundation Center for Biosustainability, Technical University of Denmark, Kogle Allé 6, 2970 Hørsholm, Denmark. ${ }^{4}$ Novo Nordisk Foundation Center for Biosustainability, Chalmers University of Technology, Kemivägen 10, 41296 Göteborg, Sweden.

\section{Acknowledgements}

The authors would like to acknowledge support from Science for Life Laboratory, the National Genomics Infrastructure, NGl, and Uppmax for providing assistance with genome sequencing and computational analysis. This work was performed in collaboration with Genomatica, Inc., and financially supported by Vetenskapsrådet, FORMAS, the Novo Nordisk Foundation and the European Research Council (Grant No. 247013).

\section{Compliance with ethical guidelines}

\section{Competing interests}

The authors declare that they have no competing interests.

Received: 25 February 2015 Accepted: 25 July 2015

Published online: 08 August 2015

\section{References}

1. Nielsen J, Jewett MC (2008) Impact of systems biology on metabolic engineering of Saccharomyces cerevisiae. FEMS Yeast Res 8(1):122-131. doi:10.1111/j.1567-1364.2007.00302.x

2. Kim IK, Roldao A, Siewers V, Nielsen J (2012) A systems-level approach for metabolic engineering of yeast cell factories. FEMS Yeast Res 12(2):228248. doi:10.1111/j.1567-1364.2011.00779.x

3. Chen Y, Nielsen J (2013) Advances in metabolic pathway and strain engineering paving the way for sustainable production of chemical building blocks. Curr Opin Biotechnol 24(6):965-972. doi:10.1016/j. copbio.2013.03.008

4. Jensen MK, Keasling JD (2014) Recent applications of synthetic biology tools for yeast metabolic engineering. FEMS Yeast Res. doi:10.1111/1567-1364.12185

5. Nielsen J, Pronk JT (2012) Metabolic engineering, synthetic biology and systems biology. FEMS Yeast Res 12(2):103. doi:10.1111/j.1567-1364.2011.00783.x

6. Flikweert MT, de Swaaf M, van Dijken JP, Pronk JT (1999) Growth requirements of pyruvate-decarboxylase-negative Saccharomyces cerevisiae. FEMS Microbiol Lett 174(1):73-79 (S0378-1097(99)00124-X [pii])

7. Schaaff I, Green JB, Gozalbo D, Hohmann S (1989) A deletion of the PDC1 gene for pyruvate decarboxylase of yeast causes a different phenotype than previously isolated point mutations. Curr Genet 15(2):75-81

8. Hohmann S, Cederberg H (1990) Autoregulation may control the expression of yeast pyruvate decarboxylase structural genes PDC1 and PDC5. Eur J Biochem 188(3):615-621

9. Hohmann S (1991) Characterization of PDC6, a third structural gene for pyruvate decarboxylase in Saccharomyces cerevisiae. J Bacteriol 173(24):7963-7969

10. Flikweert MT, Van Der Zanden L, Janssen WM, Steensma HY, Van Dijken JP, Pronk JT (1996) Pyruvate decarboxylase: an indispensable enzyme for growth of Saccharomyces cerevisiae on glucose. Yeast 12(3):247-257. doi:10.1002/(SICI)1097-0061(19960315)12:3<247:AID-YEA911>3.0.CO;2-I

11. van Maris AJ, Luttik MA, Winkler AA, van Dijken JP, Pronk JT (2003) Overproduction of threonine aldolase circumvents the biosynthetic role of pyruvate decarboxylase in glucose-limited chemostat cultures of Saccharomyces cerevisiae. Appl Environ Microbiol 69(4):2094-2099
12. van Maris AJ, Geertman JM, Vermeulen A, Groothuizen MK, Winkler AA, Piper MD et al (2004) Directed evolution of pyruvate decarboxylasenegative Saccharomyces cerevisiae, yielding a C2-independent, glucosetolerant, and pyruvate-hyperproducing yeast. Appl Environ Microbiol 70(1):159-166

13. Oud B, Flores CL, Gancedo C, Zhang X, Trueheart J, Daran JM et al (2012) An internal deletion in MTH1 enables growth on glucose of pyruvatedecarboxylase negative, non-fermentative Saccharomyces cerevisiae. Microb Cell Fact 11(1):131. doi:10.1186/1475-2859-11-131

14. Gamo FJ, Lafuente MJ, Gancedo C (1994) The mutation DGT1-1 decreases glucose transport and alleviates carbon catabolite repression in Saccharomyces cerevisiae. J Bacteriol 176(24):7423-7429

15. Blazquez MA, Gamo FJ, Gancedo C (1995) A mutation affecting carbon catabolite repression suppresses growth defects in pyruvate carboxylase mutants from Saccharomyces cerevisiae. FEBS Lett 377(2):197-200 (00145793(95)01337-7 [pii])

16. Lafuente MJ, Gancedo C, Jauniaux JC, Gancedo JM (2000) Mth1 receives the signal given by the glucose sensors Snf3 and Rgt2 in Saccharomyces cerevisiae. Mol Microbiol 35(1):161-172 (mmi1688 [pii])

17. Schulte F, Wieczorke R, Hollenberg CP, Boles E (2000) The HTR1 gene is a dominant negative mutant allele of MTH1 and blocks Snf3-and Rgt2-dependent glucose signaling in yeast. J Bacteriol 182(2):540-542. doi:10.1128/Jb.182.2.540-542.2000

18. Ozcan S, Freidel K, Leuker A, Ciriacy M (1993) Glucose uptake and catabolite repression in dominant HTR1 mutants of Saccharomyces cerevisiae. J Bacteriol 175(17):5520-5528

19. Moriya H, Johnston M (2004) Glucose sensing and signaling in Saccharomyces cerevisiae through the Rgt2 glucose sensor and casein kinase I. P Natl Acad Sci USA 101(6):1572-1577. doi:10.1073/pnas.0305901101

20. Lakshmanan J, Mosley AL, Ozcan S (2003) Repression of transcription by Rgt1 in the absence of glucose requires Std1 and Mth1. Curr Genet 44(1):19-25. doi:10.1007/s00294-003-0423-2

21. Polish JA, Kim JH, Johnston M (2005) How the Rgt 1 transcription factor of Saccharomyces cerevisiae is replated by glucose. Genetics 169(2):583-594. doi:10.1534/genetics.104.034512

22. Kruckeberg AL, Bisson LF (1990) The HXT2 gene of Saccharomyces cerevisiae is required for high-affinity glucose transport. Mol Cell Biol 10(11):5903-5913

23. Kim KS, Rosenkrantz MS, Guarente L (1986) Saccharomyces cerevisiae contains two functional citrate synthase genes. Mol Cell Biol 6(6):1936-1942

24. Vidal M, Buckley AM, Hilger F, Gaber RF (1990) Direct selection for mutants with increased $\mathrm{K}+$ transport in Saccharomyces cerevisiae. Genetics 125(2):313-320

25. Cole C, Barber JD, Barton GJ (2008) The Jpred 3 secondary structure prediction server. Nucleic Acids Res 36:W197-W201. doi:10.1093/Nar/Gkn238

26. Roy A, Kucukural A, Zhang Y (2010) I-TASSER: a unified platform for automated protein structure and function prediction. Nat Protoc 5(4):725738. doi:10.1038/nprot.2010.5

27. Entian K-D, Kötter P (2007) 25 Yeast genetic strain and plasmid collections. In: Ian S, Michael JRS (eds) Methods in microbiology. Academic, USA, pp 629-666

28. Kim JH, Brachet $V$, Moriya $H$, Johnston M (2006) Integration of transcriptional and posttranslational regulation in a glucose signal transduction pathway in Saccharomyces cerevisiae. Eukaryot Cell 5(1):167-173. doi:10.1128/EC.5.1.167-173.2006

29. Chen Y, Zhang Y, Siewers V, Nielsen J (2015) Ach1 is involved in shuttling mitochondrial acetyl units for cytosolic C2 provision in Saccharomyces cerevisiae lacking pyruvate decarboxylase. FEMS Yeast Res 15(3). doi:10.1093/femsyr/fov015

30. Flikweert MT, van Dijken JP, Pronk JT (1997) Metabolic responses of pyruvate decarboxylase-negative Saccharomyces cerevisiae to glucose excess. Appl Environ Microbiol 63(9):3399-3404

31. Gey U, Czupalla C, Hoflack B, Rodel G, Krause-Buchholz U (2008) Yeast pyruvate dehydrogenase complex is regulated by a concerted activity of two kinases and two phosphatases. J Biol Chem 283(15):9759-9767. doi:10.1074/jbc.M708779200

32. Bowman SB, Zaman Z, Collinson LP, Brown AJ, Dawes IW (1992) Positive regulation of the LPD1 gene of Saccharomyces cerevisiae by the HAP2/ HAP3/HAP4 activation system. Mol Gen Genet 231(2):296-303 
33. Reifenberger E, Boles E, Ciriacy M (1997) Kinetic characterization of individual hexose transporters of Saccharomyces cerevisiae and their relation to the triggering mechanisms of glucose repression. Eur J Biochem 245(2):324-333

34. Ozcan S, Johnston M (1995) Three different regulatory mechanisms enable yeast hexose transporter (HXT) genes to be induced by different levels of glucose. Mol Cell Biol 15(3):1564-1572

35. Gancedo JM (2008) The early steps of glucose signalling in yeast. FEMS Microbiol Rev 32(4):673-704. doi:10.1111/j.1574-6976.2008.00117.x

36. Ruiz-Roig C, Vieitez C, Posas F, de Nadal E (2010) The Rpd3L HDAC complex is essential for the heat stress response in yeast. Mol Microbiol 76(4):1049-1062. doi:10.1111/j.1365-2958.2010.07167.x

37. Knott SR, Viggiani CJ, Tavare S, Aparicio OM (2009) Genome-wide replication profiles indicate an expansive role for $\mathrm{Rpd} 3 \mathrm{~L}$ in regulating replication initiation timing or efficiency, and reveal genomic loci of Rpd3 function in Saccharomyces cerevisiae. Genes Dev 23(9):1077-1090. doi:10.1101/ gad.1784309

38. Yi C, Ma M, Ran L, Zheng J, Tong J, Zhu J et al (2012) Function and molecular mechanism of acetylation in autophagy regulation. Science 336(6080):474-477. doi:10.1126/science.1216990

39. Kurdistani SK, Grunstein M (2003) Histone acetylation and deacetylation in yeast. Nat Rev Mol Cell Biol 4(4):276-284. doi:10.1038/nrm1075

40. Takahashi H, McCaffery JM, Irizarry RA, Boeke JD (2006) Nucleocytosolic acetyl-coenzyme $A$ synthetase is required for histone acetylation and global transcription. Mol Cell 23(2):207-217. doi:10.1016/j. molcel.2006.05.040

41. Erdeniz N, Mortensen UH, Rothstein R (1997) Cloning-free PCR-based allele replacement methods. Genome Res 7(12):1174-1183

42. Guldener U, Heck S, Fielder T, Beinhauer J, Hegemann JH (1996) A new efficient gene disruption cassette for repeated use in budding yeast. Nucleic Acids Res 24(13):2519-2524 (6w0051 [pii])
43. Gietz RD, Woods RA (2002) Transformation of yeast by lithium acetate/single-stranded carrier DNA/polyethylene glycol method. Methods Enzymol 350:87-96

44. Thompson JR, Register E, Curotto J, Kurtz M, Kelly R (1998) An improved protocol for the preparation of yeast cells for transformation by electroporation. Yeast 14(6):565-571. doi:10.1002/ (SICI) 1097-0061(19980430)14:6<565:AID-YEA251>3.0.CO;2-B

45. Solis-Escalante D, Kuijpers NG, Bongaerts N, Bolat I, Bosman L, Pronk JT et al (2013) amdSYM, a new dominant recyclable marker cassette for Saccharomyces cerevisiae. FEMS Yeast Res 13(1):126-139. doi:10.1111/1567-1364.12024

46. Verduyn C, Postma E, Scheffers WA, Van Dijken JP (1992) Effect of benzoic acid on metabolic fluxes in yeasts: a continuous-culture study on the regulation of respiration and alcoholic fermentation. Yeast 8(7):501-517. doi:10.1002/yea.320080703

47. McKenna A, Hanna M, Banks E, Sivachenko A, Cibulskis K, Kernytsky A et al (2010) The Genome Analysis Toolkit: a MapReduce framework for analyzing next-generation DNA sequencing data. Genome Res 20(9):12971303. doi:10.1101/gr.107524.110

48. Teste MA, Duquenne M, Francois JM, Parrou JL (2009) Validation of reference genes for quantitative expression analysis by real-time RT-PCR in Saccharomyces cerevisiae. BMC Mol Biol 10:99. doi:10.1186/1471-2199-10-99

49. Edgar RC (2004) MUSCLE: multiple sequence alignment with high accuracy and high throughput. Nucleic Acids Res 32(5):1792-1797. doi:10.1093/nar/gkh340

50. Kiefer F, Arnold K, Kunzli M, Bordoli L, Schwede T (2009) The SWISSMODEL repository and associated resources. Nucleic Acids Res 37(Database issue):D387-D392. doi:10.1093/nar/gkn750

\section{Submit your next manuscript to BioMed Central and take full advantage of:}

- Convenient online submission

- Thorough peer review

- No space constraints or color figure charges

- Immediate publication on acceptance

- Inclusion in PubMed, CAS, Scopus and Google Scholar

- Research which is freely available for redistribution

Submit your manuscript at

www.biomedcentral.com/submit

C BioMed Central 